\title{
Acute Coronary Syndromes in Pregnant Women: Diagnosis, Potential Causes, Roadmap in Treatment, Experimental Studies and Hypotheses
}

Author:

Ertunc Simdi, Doctor Of Medicine

Camoluk Hospital, Turkey

Institutional e- mail: ertunc.simdi@saglik.gov.tr

\section{Summary:}

In this study, we wanted to discuss the acute coronary syndromes in the pregnant womens which are very important for life ofthe fetus. Spontaneous coronary artery dissection and management, which is the most common cause of acute coronary syndrome in pregnant women is also discussed. About 300 articles were examined and 35 articles which published in high quality journals with high level of evidence were chosen and this review was prepared.

Keywords: Acute Coronary Syndrome, STEMI, Spontaneous Coronary Artery Dissection, NSTEMI, Vasospastic Angina, Preeclampsia, Eclampsia, Systemic Lupus Erythromatosus

\section{METHOD}

We have prepared this review due to the fact that there are very few articles on this subject, the subject needs a more comprehensive explanation and the subject is unknown. Spontaneous coronary artery dissection and management, which is the most common cause of acute coronary syndrome in pregnant women, is discussed. MeSH terms " (Myocardial infarction OR Acute Coronary Syndromes OR STEMI OR SYSTEM) AND Pregnancy ", " (Pregnancy OR Pregnant) AND (Myocardial Infarction OR Acute Coronary Syndromes OR STEMI OR NSTEMI) ", PAMI, P-SCAD between 2012 and 2019 also PubMed and Google Academic articles were searched.

\section{INTRODUCTION}

In this study, we wanted to discuss both maternal life and acute coronary syndromes which are very important for fetus life. First of all, we have mentioned two terms that are important for this subject. Myocardial infarction in pregnancy is called 'pregnancy-associated myocardial infarction (PAMI) if it is associated with pregnancy. Coronary dissection associated with pregnancy is called Pregnancyassociated spontaneous coronary artery dissection (P-SCAD) '. In this article, acute coronary syndromes seen during pregnancy are discussed.

\section{DISCUSSION}

Epidemiology

Increased rates of spontaneous coronary artery dissection and procoagulant status in pregnant women pave the way for myocardial infarction. Myocardial infarction, which is the most common of acute coronary syndromes in pregnant women, occurs once every 10,000 pregnancy. It is seen twice 
as much as a woman who is not pregnant at the same age. (1) In pregnant women, infarction occurs clinically between 31-40 years, median age 35 years and last trimester. (2) Most of the cases are diagnosed as postmortem. (3) Myocardial infarction seen in pregnant women is a cardiac event with high mortality. In 1985, mortality ranged from 7 to 21 percent. (4) However, the mortality rate is between 5 and 37 percent. It is an increasing cause of indirect maternal death in the United States compared to European countries. [5] In contrast, sudden cardiac death syndrome, peripartum cardiomyopathy (PPCM), aortic dissection and myocardial infarction (MI) are the most common maternal deaths in the UK during 2006-2008. (6)

\section{Etiology}

The most common cause of acute coronary syndromes during pregnancy is coronary artery dissection. This is generally thought to be induced by pregnancy. The second most common cause is atherosclerosis. (7) The most common coronary artery responsible is the left anterior descending coronary artery (LAD). (8) The possibility of dissection should be considered in the approach to acute coronary syndromes during pregnancy. According to case series, 43 percent of women had spontaneous coronary artery dissection, 27 percent had atherosclerosis, 17 percent had coronary thrombosis, 3 percent had Takatsubo syndrome, and 9 percent had normal coronary arteries. $(9,10$, 11) It is said that coronary vasospasm due to hyperthyroidism may play a role in the etiology. Especially hypertension and smoking are additional risk factors. (12)

In the etiology, high levels of progesterone occur, causing structural changes in vessel wall collagen. Ergometrin, which was given postpartum hemorrhage control, was also associated with coronary vasospasm and ischemia in the pathophysiology of PAMI. This explains the pregnancy-related myocardial infarction that may be seen in the postpartum period. (13)

SCAD, real lumen tight It is defined as intramural hematoma within the coronary artery. This hematoma may create a condition that compresses the arterial lumen, reduces antegrade blood flow, and then causes myocardial ischemia or infarction $(14,15,16)$. This is the most common cause of $\mathrm{MI}$ due to pregnancy, $24 \%$ of all women under the age of 50 are reported to be the cause of MI. This is predominantly seen in young healthy women who do not have a conventional risk factor for coronary artery or heart disease. SCAD during peripartum is defined as spontaneous coronary artery dissection due to pregnancy. Spontaneous coronary artery dissection (SCAD) is an increasing cause of acute coronary syndromes (ACS), predominantly affecting middle-aged women. SCAD is defined as the separation of coronary artery walls and forming false lumens for non-traumatic and non-iatrogenic reasons. (17). Observational studies have reported a high prevalence of coronary vascular anomalies, particularly fibromuscular dysplasia (FMD), and the prevalence of low incidence of atherosclerosis. PHACTR1 / EDN1 is a risk locus that serves as a potential enhancer for the endothelin-1 (EDN1) gene for many vascular diseases, including FMD and coronary artery disease. The PHACTR gene is predominantly involved in actin reorganization in the heart muscle and brain nerve cells, as well as tubule formation and endothelial cell protection. Polymorphisms in the A allele often affect women. Perhaps this may be the reason for spontaneous arterial dissection, especially in women. (18) After spontaneous coronary artery dissection, there was cardiogenic shock in the mother, bradycardia in the fetus and cesarean section in the cardiac catheterization room. The women in this case survived coronary bypass graft surgery(19).

\section{Diagnosis}

When making the diagnosis of acute coronary syndrome during pregnancy, non-invasive methods should be used which poses the least risk to the fetus. The accuracy of exercise electrocardiography in the diagnosis of coronary artery disease is lower in women than in men and fetal bradycardia during maximum exercise has been reported in healthy women (20). Therefore, submaximal exercise 
protocol is recommended during fetal monitoring for the evaluation of ischemic myocardial disease in pregnancy $(21,22)$. Nuclear imaging should not be performed, especially in the first trimester, especially because of the risk of teratogenesis. In the second and third trimesters, nuclear imaging may lead to intrauterine growth retardation, central nervous system abnormalities, and perhaps an increased risk of malignancy. Stress echocardiography is a reasonable option to assess left ventricular function and ischemia.

Exposure below 10 radios during angiography is considered to be low risk, but exposure higher than 15 radios carries a high risk of fetal damage (23). Cardiac catheterization should only be used during pregnancy or when other diagnostic methods are not sufficient or appropriate. It has been reported that $45 \%$ of patients in 859 acute myocardial infarction case series underwent cardiac catheterization during pregnancy and postpartum. $81 \%$ of these patients underwent angioplasty, stent placement, or cardiopulmonary bypass. (24)

75/100 of the women with myocardial infarction have STEMI as the type of acute coronary syndrome and half of the women have an ejection fraction of less than 40 percent at admission. Especially the anterior wall is affected. Moderate CK and CK-MB increase is seen during pregnancy. However, troponin increase is not seen. Therefore, troponin is useful in the diagnosis. Even with preeclampsia, isolated troponin elevation indicates ischemia. (25) Preeclampsia, pulmonary embolism and aortic dissection should be considered in the differential diagnosis. ST segment depression that mimics myocardial ischemia during cesarean section but is not associated with wall motion abnormalities has also been reported (26).

As in non-pregnant patients, the diagnosis of acute myocardial infarction in pregnant patients is confirmed primarily by changes in electrocardiography and cardiac enzyme levels. Left or right axis deviation in normal pregnancy, a small Q wave in lead III, T wave inversion, or an increased R / S ratio in V1 and V2 may make electrocardiographic diagnosis of ischemia more difficult.

Cell-derived microparticles (MPs), which foresees prothrombotic status in pregnant women in the first trimester, can be used in future pregnant women. However, more research is needed for this. (27)

\section{Treatment}

The management of the patient requires a multidisciplinary approach, starting from the emergency department physician, including the obstetrician, cardiologist and anesthesiologist. Ideally, patient maternal and fetal monitoring as well as extensive obstetric cares should be treated in an intensive care unit. The optimal drug regimen for pregnant women with ischemic heart disease or acute myocardial infarction is unknown. There is important evidence to support the use of salicylate, betablockers, nitroglycerin, calcium antagonists and heparin during pregnancy.

Salicylates pass through the placenta and enter the fetal circulation. High-dose acetylsalicylic acid has been associated with fetal complications such as mortality, intrauterine growth retardation, salicylate poisoning, bleeding abnormalities, neonatal acidosis and premature closure of the ductus arteriosus (28). Adverse effects reported in the mother include anemia, bleeding, prolonged pregnancy and prolonged labor. Generally, a low dose of acetylsalicylic acid (40 mg / day to $150 \mathrm{mg} /$ day) during pregnancy does not harm the fetus and should be used in patients with known coronary artery disease.

Because of its large molecular size, heparin does not cross the placenta and is not teratogenic. Therefore, the preferred anticoagulant during pregnancy. Chronic use of high-dose subcutaneous heparin requires monitoring factor Xa levels. Heparin should be discontinued 24 hours before elective 
delivery; In case of spontaneous delivery, protamine sulfate may be required to reduce the risk of bleeding and to provide safe local and epidural anesthesia (29). After sufficient homeostasis is achieved, heparin can be continued after birth.

Little is known about the safety of other antiplatelet agents during pregnancy, including clopidogrel, ticlopidine, and platelet glycoprotein IIb / IIla inhibitors. The limited information published consists of case reports describing the use of clopidogrel at the end of 18 weeks (30).

Primary $\mathrm{PCl}$ (primary percutaneous coronary intervention) should be considered as a priority instead of thrombolytic therapy in pregnancy. Therefore, the possibility of dissection should be kept in mind in the treatment of a pregnant woman presenting with acute coronary syndrome. In contrast, primary percutaneous intervention cannot be performed and if possible, thrombolytic can be performed by considering the profit-loss ratio if we are moving away from the possibility of dissection on MRI angiography.

As a general rule, thrombolytics do not exceed plesenta, but Leonard's 28-series literature review in 2006 found that 2 fetuses died due to direct thrombolytic complications. [31] research is more secure because it focuses on thrombolytic. (32) However, thrombolytics are generally considered nonteratogenic. Percutaneous coronary intervention should not be performed, especially in the first trimester. Because ionized radiation is teratogen. However, when an infarction is detected in the first trimester, thrombolytic use should also be taken into consideration because spontaneous artery dissection is the most common cause of myocardial infarction in pregnant women. If a myocardial infarction is detected in the 2 nd or 3 rd trimester, $\mathrm{PCl}$ can be tried. (33) This procedure should be done from the radial artery.

Fetal survival is mostly associated with maternal stabilization and survival. In a systematic review of cardiovascular surgery cases published between 1984 and 1996, Weiss et al. Reported that nonpregnant patients had higher mortality (6\%) than non-pregnant patients; however, most of the deaths occurred in patients with aortic artery dissection or pulmonary embolism. (34)

The timing of surgery is effective on fetal outcome. Surgery may be preferred in the early period of the second trimester. Late second trimester or early third trimester operations should be avoided, if possible, to minimize the risk of preterm labor. If the gestational week of the fetus is more than 28 weeks, the baby should be delivered immediately before and during the same operation.

When we look at the potential therapies, there was an increase in the ejection fraction when the TREG cells of the pregnant and fetus, which were considerably increased during pregnancy, were injected into patients with myocardial infarction, whose TREG cells were significantly reduced. (35)

\section{CONCLUSION}

Myocardial infarction in pregnancy is a high mortality rate that is overlooked. Diagnosis, treatment and potential treatment methods are explained. In this article myocardial infarction during pregnancy is discussed. In the diagnosis of chest pain in a pregnant woman should be monitored and further investigations should be done. When we look at the etiology, coronary artery dissection comes first. 75/100 of the women with myocardial infarction have STEMI as the type of acute coronary syndrome and half of the women have an ejection fraction of less than 40 percent at admission. Especially the anterior wall is affected. ECG and especially troponin are useful in diagnosis. This examination is not useful since CK-MB is already mildly elevated during pregnancy. Minimally invasive methods should be used in treatment. Primary invasive routes should be preferred. Potentially easy, diagnostic and therapeutic research is needed. For fetal stabilization, it is very important for maternal stabilization. 


\section{Abbreviations}

STEMI: ST Elevation Acute Myocardial Infarction

NSTEMI: Non ST Segment Elevation Myocardial Infarction

P-SCAD: Pregnancy spontan coronary artery dissection

PAMI: Pregnancy associated myocardial infarction

1)Paratz ED, Kao C, Maclsaac Al, Somaratne J, Whitbourn R., Evolving management and improving outcomes of pregnancy-associated spontaneous coronary artery dissection (P-SCAD): a systematic review, Int J Cardiol Heart Vasc. 2018 Jan 2;18:1-6. doi: 10.1016/j.ijcha.2017.12.001. eCollection 2018 Mar.

2)Eric J Gartman, The use of thrombolytic therapy in pregnancy, Obstet Med.( 2013) Sep;6(3):105-111

3) Smilowitz, Nathaniel R. et al, Acute Myocardial Infarction During Pregnancy and the Puerperium: Experiences and Challenges From Southern India., Mayo Clinic Proceedings, Volume 94, Issue 5, May (2019), Pages 918-919

4) Smilowitz, Nathaniel R. et al., Acute Myocardial Infarction During Pregnancy and the Puerperium in the United States, Mayo Clinic Proceedings, Volume 93, Issue 10, 1404 - 1414., October (2018), Volume 93, Issue 10, Pages 1404-1414

5) Pierce, T., Hovnanian, M., Hedgire, S. et al. Imaging of Cardiovascular Disease in Pregnancy and the Peripartum Period. Curr Treat Options Cardio Med 19, 94 (2017) doi:10.1007/s11936-017-0593-8

6)Vera Regitz-Zagrosek Jolien W Roos-Hesselink Johann Bauersachs Carina Blomström-Lundqvist Renata Cífková Michele De Bonis, 2018 ESC Guidelines for the management of cardiovascular diseases during pregnancy, European Heart Journal, Volume 39, Issue 34, 7 September (2018), 31653241, https://doi.org/10.1093/eurhearti/ehy340

7) .Rashid HN, Wong DT, Wijesekera H, Gutman SJ, Shanmugam VB, Gulati R, Malaipan Y, Meredith IT, Psaltis PJ. Incidence and characterisation of spontaneous coronary artery dissection as a cause of acute coronary syndrome-a single-centre Australian experience. (2016) Int J Cardiol.; 202:336-338. doi: 10.1016/j.ijcard.2015.09.072.

8)Havakuk O1, Goland S1, Mehra A1, Elkayam U2.,Pregnancy and the Risk of Spontaneous Coronary Artery Dissection: An Analysis of 120 Contemporary Cases.Circ Cardiovasc Interv. (2017) Mar;10(3). pii: e004941. doi: 10.1161/CIRCINTERVENTIONS.117.004941.

9) Pierce, T., Hovnanian, M., Hedgire, S. et al. Imaging of Cardiovascular Disease in Pregnancy and the Peripartum Period. Curr Treat Options Cardio Med 19, 94 (2017) doi:10.1007/s11936-017-0593-8

10) Wu P, Gulati M, Kwok CS, Wong CW, Narain A, O'Brien S, Chew-Graham CA, Verma G, Kadam UT, Mamas MA. Preterm Delivery and Future Risk of Maternal Cardiovascular Disease: A Systematic Review and Meta-Analysis. (2018) J Am Heart Assoc. Jan 15;7(2):e007809. doi:

10.1161/JAHA.117.007809. PMID: 29335319; PMCID: PMC5850169.

11) Papsdorf M., Wöckel A., Kranke P. Risikoschwangerschaft, Schwangerschaftserkrankungen und pathologische Geburt. In: Kranke P. (eds) (2017) Die geburtshilfliche Anästhesie. Springer, Berlin, Heidelberg.

12)Honigberg MC, Scott NS, Pregnancy-Associated Myocardial Infarction,Curr Treat Options Cardiovasc Med. (2018) Jun 19;20(7):58. doi: 10.1007/s11936-018-0655

13) Gibson P1, Narous M2, Firoz T3, Chou D4, Barreix M4, Say L4, James M, Incidence of myocardial infarction in pregnancy: a systematic review and meta-analysis of population-based studies, European Heart Journal - Quality of Care and Clinical Outcomes, Volume 3, Issue 3, 1 July (2017), Pages 198-207, https://doi.org/10.1093/ehjqcco/qcw060 
14) Ravichandran, Jeganathan et al ,High-Sensitivity Cardiac Troponin I Levels in Normal and Hypertensive Pregnancy. The American Journal of Medicine, Volume 132, March (2019), Issue 3, 362 $-366$

15) Wang, L., Yang, J., Zheng, J., \& Gu, X. Acute myocardial infarction in pregnancy: spasm caused by hyperthyroidism? Journal of International Medical Research. (2019)

https://doi.org/10.1177/0300060519837833

16) Vera Regitz-Zagrosek, Myocardial infarction in pregnancy: how frequent, how fatal?, European Heart Journal - Quality of Care and Clinical Outcomes, Volume 3, Issue 3, July (2017), Pages 171-172, https://doi.org/10.1093/ehjqcco/qcx006

17)DavidAdlamDPhila, Timothy M.Olson MDb, NicolasCombaretMDc, Jason C.Kovacic MD, PhD, Association of the PHACTR1/EDN1 Genetic Locus With Spontaneous Coronary Artery Dissection Journal of the American College of Cardiology, Volume 73, Issue 1, 8-15 January (2019), Pages 67-69, https://doi.org/10.1016/i.jacc.2018.09.085

18)VarunSundaramMD, Sunit-PreetChaudhryMD, Yogesh N.V.ReddyMD, Chris T.LongeneckerMD, James C.FangMD, Current Treatment Options in Cardiovascular Medicine; Sep(2017), Vol. 19 Issue 9, p1-7, 7p, https://doi.org/10.1016/j.amjcard.2015.03.027

19) Alyson J. McGregor, Rebecca Barron, Karen Rosene-Montella, The pregnant heart: cardiac emergencies during pregnancy, March 06, (2015) DOI:https://doi.org/10.1016/j.ajem.2015.02.046

20) Adam, Karolina. "Pregnancy in Women with Cardiovascular Diseases." Methodist (2017) DeBakey cardiovascular journal vol. 13,4 209-215. doi:10.14797/mdcj-13-4-209

21) Kolovou, Genovefa; Kolovou, Vana; Koutelou, Maria; Mavrogeni, Sophie , Atherosclerotic and Non-Atherosclerotic Coronary Heart Disease in Women, (2015) Current Medicinal Chemistry, Volume 22, Number 31, pp. 3555-3564(10)

22) Rose, Emily et al., Pregnancy-Related Spontaneous Coronary Artery Dissection: A Case Series and Literature Review (2017) Journal of Emergency Medicine, Volume 52, Issue 6, 867 - 874

23) Spencer, SPE, Lowe, SA. Ergometrine for postpartum hemorrhage and associated myocardial ischemia: Two case reports and a review of the literature. (2019) Clin Case Rep.; 00: 1- 10. https://doi.org/10.1002/ccr3.2516

24)Elkayam U. Pregnancy and cardiovascular disease In: Zipes DP, Libby P, Bonow RO, Braunwald E, eds Braunwald's Heart Disease: A Textbook of Cardiovascular Medicine. Philadelphia: (2014) Elsevier Inc; pp. 1965-84.

25) Ismail, Sahar et al. "ST-elevation acute myocardial infarction in pregnancy: 2016 update." Clinical cardiology vol. 40,6 (2017): 399-406. doi:10.1002/clc.22655

26) Liu, Hui Ding, Yi-Ling Huang, Jian Two cases of acute aortic dissection following preeclampsia in non-Marfan patients AU , (2012) Chinese medical journal- 10.3760/cma.j.issn.0366-6999.2012.11.043

27) Jaume Alijotas-Reig, Carles Palacio-Garcia, Elisa Llurba, Miquel Vilardell-Tarres, Cell-derived microparticles and vascular pregnancy complications: a systematic and comprehensive review, (2012) Fertility and Sterility, Volume 99, Issue 2, Pages 441-449

28) LeFevre ML, on behalf of the U.S. Preventive Services Task Force. Low-Dose Aspirin Use for the Prevention of Morbidity and Mortality From Preeclampsia: U.S. Preventive Services Task Force Recommendation Statement. (2014)Ann Intern Med.;161:819-826. doi: https://doi.org/10.7326/M14-1884

29)Olausson N1, Mobarrez F, Wallen H, Westerlund E, Hovatta O, Henriksson P. Microparticles reveal cell activation during IVF - a possible early marker of a prothrombotic state during the first trimester.Thromb Haemost. (2016) Aug 30;116(3):517-23. doi: 10.1160/TH15-12-0970.

30) Borhart, Joelle et al. Cardiovascular Emergencies in Pregnancy, (2019) Emergency Medicine Clinics, Volume 37, Issue 2, 339 - 350 
31) Mehra, A., Muadi, G., Reddy, P. and Elkayam, U. Catheter - Based Interventions in Women with Heart Disease During Pregnancy. In Cardiac Problems in Pregnancy, U. Elkayam (Ed.). (2019). doi:10.1002/9781119409861.ch27

32) Pregnancy-Associated Acute Myocardial Infarction A Review of Contemporary Experience in 150 Cases Between 2006 and 2011 Uri Elkayam, Sawan Jalnapurkar, Mohamad N. Barakkat, Nudrat Khatri, Angela J. Kealey, Anil Mehra, and Arie Roth Originally published, 22 Apr (2014) Circulation https://doi.org/10.1161/CIRCULATIONAHA.113.002054Circulation. 2014;129:1695-1702

33) Rick A. Nishimura, Catherine M. Otto, Robert O. Bonow, Blase A. Carabello, John P. Erwin, Robert A. Guyton, Patrick T. O'Gara, Carlos E. Ruiz, Nikolaos J. Skubas, Paul Sorajja, Thoralf M. Sundt, James D. Thomas J Am Coll Cardiol. (2014) Jun, 63 (22) e57-e185.

34)Lee, R.,\& Carr, D. (Pregnancy-associated spontaneous coronary artery dissection (PASCAD): An etiology for chest pain in the young peripartum patient. (2018). CJEM, 20(S2), S64-S69. doi:10.1017/cem.2018.9

35)Paracrine effect of regulatory T cells promotes cardiomyocyte proliferation during pregnancy and after myocardial infarction, Serena Zacchigna,Valentina Martinelli, Silvia Moimas , Andrea Colliva, Marco Anzini, Andrea Nordio, Nat Commun. (2018); 9: 2432. Published online 2018 Jun 26.

doi:10.1038/s41467-018-04908-z 